\title{
The significance of central venous pressure and cardiac output measurements in shock
}

\author{
J. F. RIORDAN* \\ G. WALTERS \\ W. D. S. McLay
}

\author{
New Cross Hospital, Wolverhampton
}

\begin{abstract}
Summary
The central venous pressure changes which occur in shock syndromes vary with the aetiology and other factors.

Two methods of measuring CVP have given different results, and their advantages and disadvantages are discussed, together with an appraisal of the diagnostic and therapeutic value of such measurements.
\end{abstract}

The CVP changes produced by transfusion are related to changes in cardiac output and the value of the latter as a guide to treatment is discussed. The relationship of these measurements to clinical signs is emphasized.

\section{Introduction}

It has been shown during the last 40 years or so, first in the laboratory and later in patients, that a fall in cardiac output is an important factor in the progressive decline of the shock state. There is currently a good deal of emphasis on the role of changes in the microcirculation, but a low cardiac output is still the dominant factor in most patients with shock and, logically, treatment must be directed towards raising it.

The cause of the lowered cardiac output varies with the aetiology, but one important determinant of cardiac output is the central venous pressure (CVP). The value of CVP measurements as an indication for, and to control, therapeutic measures to raise the cardiac output will be discussed in detail, but only brief reference will be made to high cardiac output states in which the output is probably not the limiting factor.

The central venous pressure is the pressure within the great veins returning blood to the heart. It depends mainly on the blood volume, the venous tone and the cardiac efficiency. Alteration in any one of these will tend to alter the CVP unless there is an opposing alteration in one of the others. Thus, a decrease in blood volume will lower the CVP if the other factors do not change, but if at the same time

\footnotetext{
*Present address: Central Middlesex Hospital, Park Royal, London, N.W.10.
}

there is an increase in venous tone or if cardiac $\vec{\omega}$ efficiency is decreased, CVP may remain unchanged. Obviously, if a decrease in blood volume and de- $\underline{3}$ crease in venous tone occur together, as in certain pathological conditions, their effects will summate or to produce a larger fall in CVP than either alone. N Because the CVP depends on the interrelationship of of these factors they must all be considered in its interpretation.

\section{Measurement of CVP}

The CVP may be measured in shocked patients by one of two methods. The method of Borst \& $\stackrel{\text { ? }}{+}$ Molhuysen (1952) depends on careful observatiogn $\vec{\circ}$ of the external jugular vein. The vertical distanes between the top of a freely pulsating column of blood in this vein and the sternal angle is measured using a suitable gauge, and expressed in centimetres above $(+)$ or below $(-)$ the sternal angle. Alternatively, a plastic intravenous catheter may be ad- $\frac{\mathscr{Q}}{\otimes}$ vanced from a peripheral vein until its tip is in the $\varrho$ superior vena cava, and connected to a saline mano- $\overrightarrow{\vec{O}}$ meter. The CVP may again be expressed as centi- 3 metres of saline above the sternal angle. Suitable sterile disposable sets are available commercially and are now widely used.

Borst \& Molhuysen (1952) obtained very similar results with the gauge and right atrial catheterization $\frac{5}{3}$ in ten patients with heart disease, but the gauge gave results about $4 \mathrm{~cm}$ lower than the catheter in two others. We have usually found lower values with the gauge than with a central venous catheter and the $ᄋ$ quoted normal range for the latter method is higher. $\rightarrow$ The differences are sometimes relatively large, but both methods show swings in the same direction N (Fig. 1), and provided the changes are interpreted according to the respective normal ranges $(-6$ to -3 N $\mathrm{cm}$ with Borst's method; -4 to $+6 \mathrm{~cm}$ with the intra- $\mathrm{C}$ venous catheter), either method allows satisfactory control of treatment. In the following account onlyco measurements obtained with an intravenous catheter $\overparen{\Phi}$ and saline manometer will be quoted so that they $\stackrel{\mathcal{C}}{+}$ may be compared more easily with published data, $\underset{T}{T}$ most of which were obtained by this method. It is 


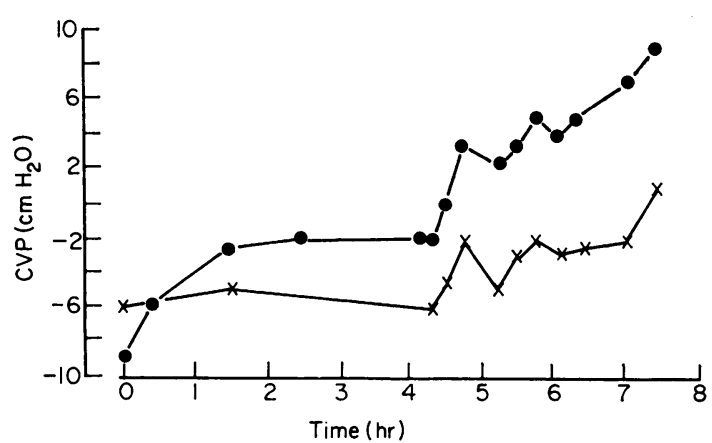

Fig. 1. Central venous pressure changes in a case of shock measured simultaneously by intravenous catheter

$(O)$ and observation of the external jugular vein $(x)$.

important to remember that different authors use different zero reference points, e.g. the mid part of chest, or $5 \mathrm{~cm}$ below the sternal angle; we use the sternal angle in a supine patient, which gives a normal range of -4 to $+6 \mathrm{~cm}$. Cardiac output measurements were obtained using a photoelectric earpiece and Coomassie Blue as described by Gabe \& Shillingford (1961). The area under the curve was calculated according to the method of Dow (1955), to eliminate false low values which may occur in low output states, due to distortion of the downslope of the curve by re-circulation (Oriol \& MacGregor, 1967).

\section{Interpretation of CVP measurements}

The term 'normal central venous pressure' is often used with reference to shocked patients. This means a CVP which falls within the range encountered in normal subjects who are at rest. Although the CVP in a shocked patient may be normal in this sense, it is of the utmost importance to decide whether or not it is adequate. For therapeutic purposes patients can be divided into three categories on the basis of CVP measurements, namely: (1) low, (2) normal, or (3) high.

The following remarks about infusion therapy in shock do not necessarily apply to cases with myocardial infarction.

Group 1. In the first group a CVP below the normal range indicates hypovolaemia or reduced venous tone or both together. It must always be regarded as inadequate in the presence of evidence of a low cardiac output and rapid infusion to raise it is indicated. This usually results in restoration of a normal circulation. Sometimes, however, persistence of the CVP-lowering factors causes it to fall again, together with a fall in cardiac output, and then repeated transfusion is required (Case 1, Fig. 2).

\section{Case 1 (Fig. 2)}

This was a patient with gas gangrene and oliguric renal failure in whom hypotension recurred five

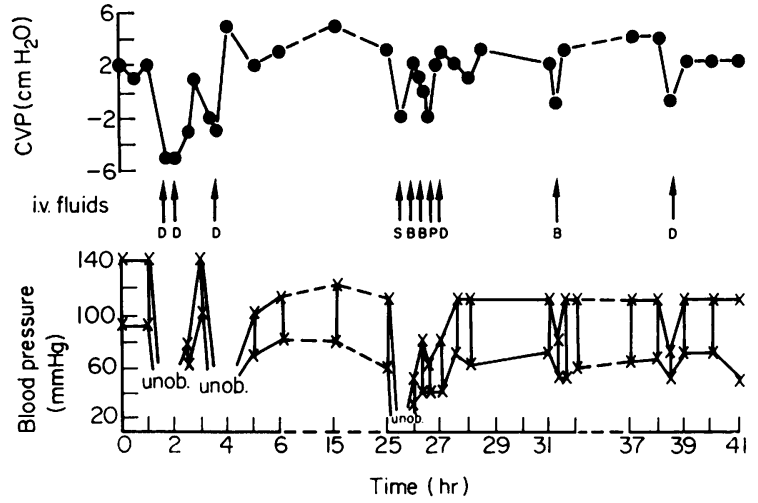

FIG. 2. Case 1. Recurrent circulatory failure in a case of gas gangrene. Each episode was accompanied by a fall in CVP and reversed by rapid transfusion to raise the CVP. The latter did not always fall to abnormally low values.

times in $30 \mathrm{hr}$, accompanied each time by a fall in CVP, a decrease in pulse volume which probably indicated a fall in cardiac output and impairment or loss of consciousness. Rapid infusion to elevate the CVP restored the blood pressure on each occasion, together with improvement in pulse volume and return of normal consciousness. Ultimately, a stable circulation was obtained.

It is worthy of note that the CVP in several of the episodes was within the normal range but was, nevertheless, clearly inadequate; these episodes thus fall within Group 2. The possibility of a CVP within the normal range being inadequate was emphasized by McGowan \& Walters (1963) who recommended infusing all such patients until the CVP reached the upper limit of normal. This worked well in the case illustrated but in some patients hypovolaemia or reduced venous tone is masked by some degree $o$ myocardial impairment, and restoring the CVP merely to the upper limit of normal may be inadequate to produce benefit. Circulatory improvement may nevertheless result from temporary elevation of the CVP to an abnormally high level (Cases 2 and 3, Figs. 3 and 4).

\section{Case 2 (Fig. 3)}

A 29-year-old man was admitted in deep coma due to barbiturate poisoning, with impalpable radial, brachial and femoral pulses, cold extremities and an unobtainable blood pressure. Cardiac output was $3.21 / \mathrm{min}$, although the CVP was normal at $+1 \mathrm{~cm}$. Plasma, $1300 \mathrm{ml}$, given in $50 \mathrm{~min}$, produced a rise in CVP to $+8 \mathrm{~cm}$ which is above our normal range, and femoral pulses became palpable. Another 450 $\mathrm{ml}$ given in $5 \mathrm{~min}$ produced a sharp rise in CVP to $+14 \mathrm{~cm}$. The drip was slowed and CVP began to fall, as it usually does in such circumstances. Within 


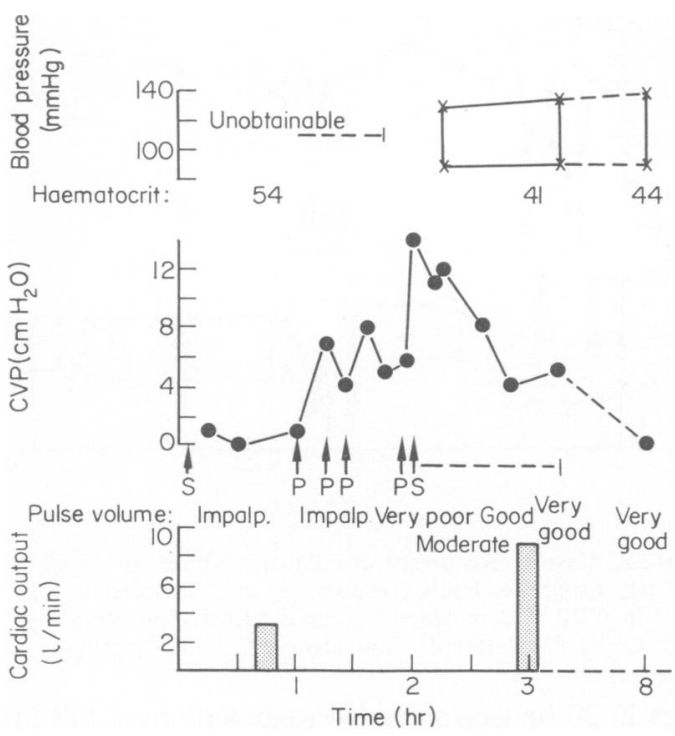

Fig. 3. Case 2. Effect of transfusion on cardiac output in a patient with acute circulatory failure due to barbiturate poisoning and a normal CVP. The subsequent fall in CVP does not indicate the need for further transfusion (cf. Fig. 2).

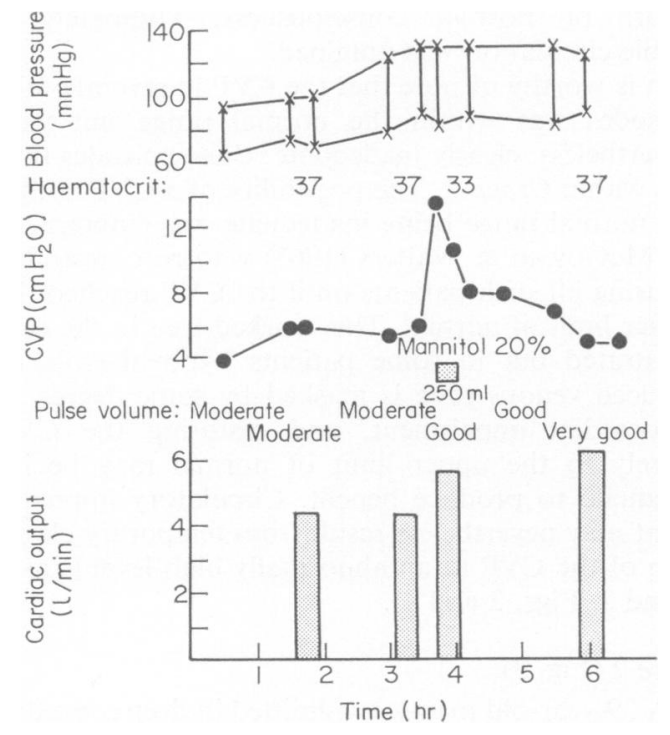

FIG. 4. Case 3. Effect on cardiac output of raising the CVP to abnormally high levels. As in Fig. 3, the later fall in CVP to former levels does not indicate the need for further transfusion.

10 min the radial pulses were of moderate volume and blood pressure was normal. Pulse volume improved further during the next $45 \mathrm{~min}$ as CVP fell progressively to $+4 \mathrm{~cm}$, by which time the pulse volume was excellent and cardiac output $8 \cdot 8 \mathrm{l} / \mathrm{min}$.
CVP fell to zero overnight, but the circulation remained satisfactory as judged by the pulse volume and arterial blood pressure.

This effect on cardiac output of an abnormally high CVP is also illustrated by Case 3 .

\section{Case 3 (Fig. 4)}

This 55-year-old man was comatose, with respiratory arrest, $24 \mathrm{hr}$ after resection of a bronchial carcinoma, and had been treated with positive pressure ventilation (PPV) for several hours before the period shown. Pulse volume was moderate, CVP $+6 \mathrm{~cm}$ and cardiac output $4 \cdot 4 \mathrm{l} / \mathrm{min}$. But urine flow was reduced, and $90 \mathrm{~min}$ later when pulse volume and cardiac output were unchanged, $250 \mathrm{ml} 20 \%$ mannitol were given rapidly. There was a sharp rise in CVP to $+13 \mathrm{~cm}$ accompanied by a fall in haematocrit. A definite increase in pulse volume occurred and cardiac output rose to $5.71 / \mathrm{min}$. The CVP then fell, rapidly at first, as in the previous case, while the cardiac output remained up as indicated by a good pulse volume. It was $6.3 \mathrm{l} / \mathrm{min} 2 \mathrm{hr}$ later when CVP and haematocrit had returned to their original levels.

In this sort of case an optimum CVP cannot be defined because raising it temporarily allows permanent cardiac improvement so that a lower pressure then suffices. The important point is that in some cases a rapid temporary elevation of CVP is followed by sustained improvement in cardiac funce tion.

\section{The significance of pulmonary oedema}

The question arises, how high can the CVP be raised by infusion without producing pulmonary oedema? Ideally, pulmonary wedge or left atrial pressure should be measured to avoid pulmonary oedema due to overinfusion but this is usually impracticable. CVP is the best practical guide provided its limitations are appreciated. Most authors agree that pulmonary oedema is unlikely to occur due to overinfusion if a CVP of +6 to $+8 \mathrm{~cm}$, with reference to the sternal angle, is not exceeded. Indeed, as we have seen, if infusion is rapid and stops when CVP rises sharply, much higher levels may be produced temporarily with safety, and often with benefit. Even when no circulatory improvement occurs, the CVP usually falls again quite quickly in these circumstances and, in our experience, pulmonary oedema does not occur. It must be emphasized that this type of treatment must be very closely supervised, and once CVP levels of +12 to $+14 \mathrm{~cm}$ are reached, the infusion must be stopped and CVP allowed to fall. If intravenous fluid is given relatively slowly, rapid changes in CVP will not occur, and such measurements may then be much less useful in controlling infusion. 
Cases 2 and 3 demonstrate that a fall in CVP after infusion does not necessarily indicate the need for more fluid, as it did in Case 1 when it was accompanied by signs of an inadequate circulation. Changes in CVP must always be interpreted together with the other haemodynamic signs and not in isolation. We have seen pulmonary oedema follow a large volume of intravenous fluid given to maintain the CVP at an arbitrary level. This was in a 30-yearold girl with pneumonia who was admitted with a blood pressure of $65 \mathrm{mmHg}$ and barely palpable pulses. She was quickly resuscitated, and then the CVP fell to $-2 \mathrm{~cm}$, which was lower than when first seen. But as the arterial blood pressure and pulse volume were satisfactory, no further infusion was needed. Nevertheless, several more litres were given overnight to maintain the CVP at $+5 \mathrm{~cm}$, and by the following morning pulmonary oedema was present. Fortunately it cleared when fluid was stopped.

Of course, the significance of pulmonary oedema in such cases is not always easy to establish. Pulmonary oedema may occur in the presence of a normal CVP, and we have seen it in patients with non-pulmonary infection given no parenteral fluid and with normal hearts. MacLean et al. (1967) have described one case of septicaemia in which pulmonary oedema occurred in the presence of a normal pulmonary wedge pressure. The explanation may be increased pulmonary capillary permeability. One of our patients (Case 1) is particularly interesting in this respect because he required rapid infusion to maintain the circulation on five occasions despite severe pulmonary oedema which needed positive pressure ventilation to control it throughout this period (Riordan \& Walters, 1968). Cases like this are rare but when pulmonary oedema occurs with a low CVP, it is worth remembering that infection may be the cause and fluids may still be needed to combat circulatory insufficiency. PPV is probably essential in these cases.

Patients in Group 3, i.e. those with a high CVP, require a different approach. The high CVP may be present from the start or may have been produced by unsuccessful infusion. A CVP persisting at +8 $\mathrm{cm}$ or more means that fluid is unlikely to be helpful and may be harmful. Other methods of raising the cardiac output such as cardiac stimulants or vasodilators must be tried. CVP monitoring must be continued as most of these drugs lower the CVP and may lower it below the optimum. Some authors advocate routine fluid administration as an adjunct to phenoxybenzamine administration in these cases. However, fluid is often unnecessary and it is preferable to watch the CVP during treatment and give fluid as indicated. The sort of changes that occur are illustrated by Cases 4 and 5 .
Case 4 (Fig. 5)

Case 4 had cardiogenic shock following prolonged paroxysmal tachycardia, with a cardiac output of $1.9 \mathrm{l} / \mathrm{min}$ and a CVP of $+23 \mathrm{~cm}$ after version to

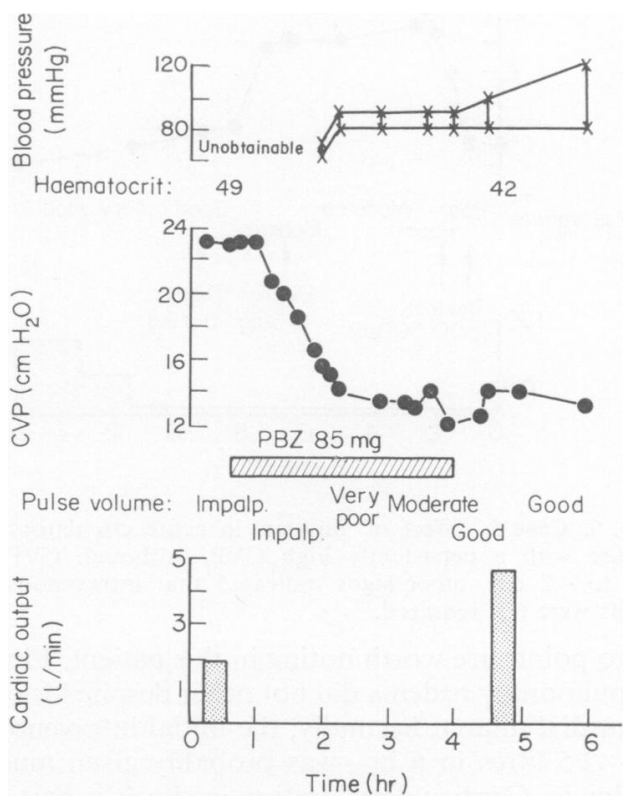

FIG. 5 Case 4. Effect of $\alpha$-adrenergic blockade with phenoxybenzamine in acute circulatory failure with a persistently high CVP. The fall in CVP was such as to contraindicate concomitant transfusion.

sinus rhythm; pulmonary oedema was present. Phenoxybenzamine given by slow intravenous infusion produced a fall in CVP to $+12 \mathrm{~cm}$, a rise in cardiac output to $4.6 \mathrm{l} / \mathrm{min}$ and concomitant clinical signs of improvement. No intravenous fluids were necessary. In another case, due to myocardial infarction, treated like this, the CVP fell to $-2 \mathrm{~cm}$. But, as the circulation was better clinically and cardiac output had risen from 2.4 to $4.0 \mathrm{l} / \mathrm{min}$, fluids were unnecessary. If, however, the CVP falls to low levels after phenoxybenzamine without a concomitant improvement in the circulation, fluids should be infused in the usual fashion.

\section{Case 5 (Fig. 6)}

This case shows similar changes in response to digoxin. Circulatory failure due to peritonitis and an old myocardial infarct persisted after 2.5 litres of fluid in $6 \mathrm{hr}$. As the CVP was only $+1 \mathrm{~cm}, 1$ litre of Dextran was given in $1 \mathrm{hr}$. The CVP rose steadily to $+10 \mathrm{~cm}$ without much improvement in the circulation and this state persisted. Digoxin intravenously produced a fall in CVP and other signs of circulatory improvement. 


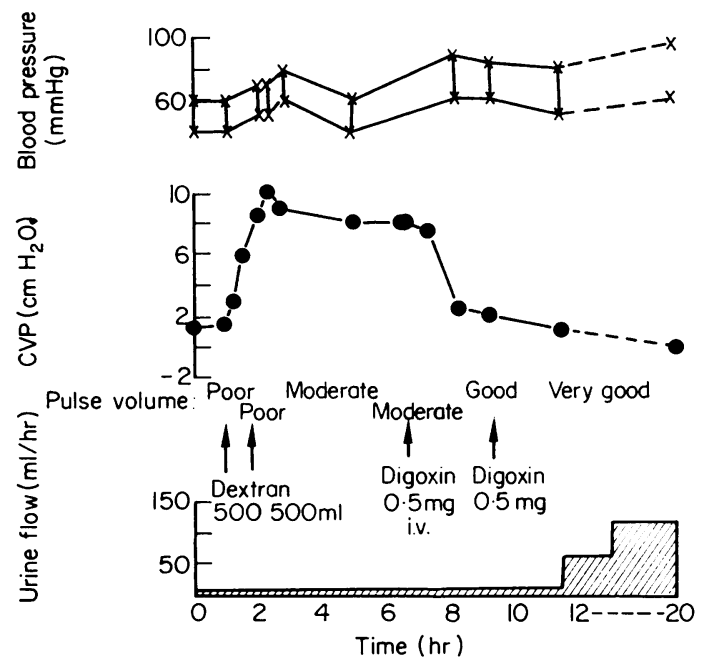

FIG. 6. Case 5. Effect of digoxin in acute circulatory failure with a persistently high CVP. Although CVP fell to $-2 \mathrm{~cm}$, other signs indicated that intravenous fluids were not required.

Two points are worth noting in this patient. First, that pulmonary oedema did not occur despite his old myocardial infarct. Secondly, the initial intravenous fluid-2.5 litres in $6 \mathrm{hr}$-was probably given much too slowly. Cardiac deterioration in shock is part of the vicious circle and the earlier shock is reversed the less likely progressive cardiac deterioration is to occur. Fluid administration, therefore, should be rapid in the early stages as illustrated above.

\section{Adequacy of cardiac output}

We have, so far, been concerned only with how CVP measurements help in applying therapy designed to raise cardiac output, and have avoided the more difficult problem of what level of cardiac output is desirable. In many cases restoration of a normal cardiac output is sufficient, but patients with sepsis appear to need outputs higher than normal to ensure recovery (Clowes, Vucinic \& Weidener, 1966). Exactly how high is difficult to judge as the final case illustrates.

\section{Case 6 (Fig. 7)}

A 37-year-old man with peritonitis. After some treatment, the blood pressure was $80 / 50 \mathrm{mmHg}$ and the CVP $-6.5 \mathrm{~cm}$, but the pulse volume was good and the cardiac output $6 \mathrm{l} / \mathrm{min}$. The urine output was low and intravenous mannitol produced a large increase in CVP and a rise in cardiac output to 10 $1 / \mathrm{min}$, which was accompanied by a rise of arterial blood pressure to normal. Subsequently, the CVP and arterial pressure fell to former levels, and cardiac output to $7 \mathrm{l} / \mathrm{min}$.

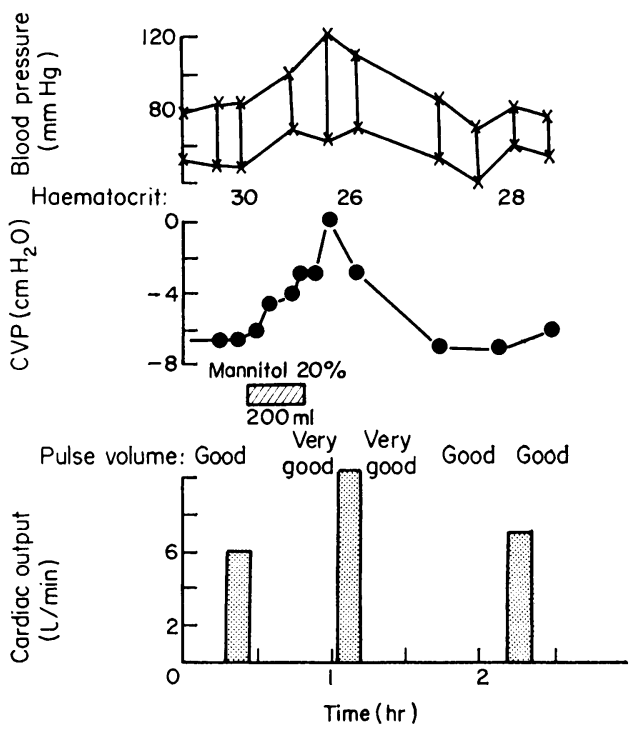

FIG. 7. Case 6. Effect of elevating CVP in a case of hypotension with a normal cardiac output.

The question arises should such a patient be transfused again to raise the CVP in the hope of maintaining a cardiac output of $10 \mathrm{l} / \mathrm{min}$ ? This is a questiog we cannot answer. In this patient, as in the other we have described, the cardiac output figures weres not available to us until 1 or 2 days afterwards, ant treatment was always based on clinical signs, particularly the pulse volume and CVP measurements. In this instance, further fluid was given until CVP rose to and remained at zero again, whereupon a sustained improvement in pulse volume, blood pressure and urine flow was obtained, and presumably, cardiac output.

Thus the clinical signs, particularly pulse volume and skin temperature and, with some reservations urine flow rate, may give useful information as to the adequacy of cardiac output. This can be reinforced by biochemical studies, e.g. reversal of a metabolic acidosis and a fall in arterial blood lactate indicate that an adequate cardiac output has been reached, but these changes may take several hours to become apparent.

Unfortunately, patients with shock due to sepsis still die, even with a high cardiac output. A hyperdynamic circulation with hypotension may be associated with a rising blood lactate and metabolic acidosis indicating defective utilization of oxygen, possibly resulting from decreased tissue perfusion due to arterio-venous shunting (Siegel, Greenspan \& del Guercio, 1967). In these patients, inadequacy of cardiac output is probably not the major limiting factor, and CVP and cardiac output measurements serve only to indicate transition to another state. 


\section{References}

Borst, J.G.G. \& Molhuysen, J.A. (1952) Exact determination of the central venous pressure by a simple clinical method. Lancet, ii, 304.

Clowes, G.H.A., Vucinic, M. \& Weidener, M.G. (1966) Circulatory and metabolic alterations associated with survival or death in peritonitis. Ann. Surg. 163, 866.

Dow, P. (1955) Dimensional relationships in dye-dilution curves from humans and dogs, with an empirical formula for certain troublesome curves. J. appl. Physiol. 7, 399.

GABE, I. \& Shillingford, J. (1961) The photo-electric earpiece technique for recording dye dilution curves. Brit. Heart J. 23, 271.
McGowan, G.K. \& Walters, G. (1963) The value of measuring central venous pressure in shock. Brit. J. Surg. 50, 821.

Maclean, L.D., Mulligan, W.G., Maclean, A.P.H. \& Duff, J.H. (1967) Patterns of septic shock in man. A detailed study of 56 patients. Ann. Surg. 166, 543.

Oriol, A. \& MCGREgor, M. (1967) Indicator-dilution methods in estimation of cardiac output in clinical shock. Amer. J. Cardiol. 20, 826.

Riordan, J.F. \& Walters, G. (1968) Pulmonary oedema in bacterial shock. Lancet, i, 719.

Siegel, J.H., GreensPan, M. \& Del Guercio, L.R.M. (1967) Abnormal vascular tone, defective oxygen transport and myocardial failure in human septic shock. Ann. Surg. 165, 504. 